\title{
A new device for measuring resting energy expenditure (REE) in healthy subjects
}

\author{
Marcella Malavolti ${ }^{a, *}$, Angelo Pietrobelli ${ }^{\text {a,b }}$, Manfredo Dugoni ${ }^{\text {a }}$, \\ Marco Poli ${ }^{a}$, Elisa Romagnoli ${ }^{a}$, Paolo De Cristofaro ${ }^{c}$, Nino C. Battistini ${ }^{a}$
}

\author{
${ }^{a}$ Applied Dietetic Technical Sciences, Modena and Reggio Emilia University, \\ Via Campi 287, 41100 Modena, Italy \\ ${ }^{\mathrm{b}}$ Paediatric Unit, Verona University Medical School, Verona, Italy \\ c Centro Regionale di Fisiopatologia della Nutrizione ASL Teramo, Italy
}

Received 20 July 2005; received in revised form 21 December 2005; accepted 22 December 2005

\section{KEYWORDS \\ Resting metabolic \\ rate; \\ Body composition; \\ Fat mass; \\ Fat free mass; \\ Healthy subjects}

\begin{abstract}
Background and aim: Lifestyle change targeted towards increasing daily resting energy expenditure (REE) is one of the cornerstones of obesity treatment. Measurements of energy expenditure and substrate utilization are essential to understanding the metabolic basis of obesity, and the physiological responses to perturbations in habitual food intake. REE is the largest part of human energy expenditure $(60-70 \%)$ and an increase or decrease in REE would have a large impact on total energy. Accurate and easy-to-use methods for measuring REE are needed, to be applied by clinicians in daily clinical settings to assess the validity of a new instrument to estimate REE in normal weight, healthy adults.

Methods: Ninety-nine subjects (52 females and 47 males) (mean \pm SD, age $38 \pm 14$ years; body mass index (BMI) $23 \pm 3 \mathrm{~kg} / \mathrm{m}^{2}$ ) were tested. REE was assessed using a Sensor Medics Vmax metabolic cart with a ventilated canopy and with the SenseWear armband. Body composition, percentage fat mass (\%FM) and percentage fat free mass (\%FFM) were assessed by skinfold thickness measurements (SF), bioelectrical impedance analysis (BIA) and air displacement plethysmography (BODPOD).

Results: No significant difference was found among measurements of FFM using the three different techniques. Both SenseWear and Sensor Medics Vmax showed a high correlation, $r=0.42$ and $r=0.40(p<0.0001)$ respectively, with BMI. No significant difference was found in mean REE between SenseWear $(1540 \pm 280 \mathrm{kcal} / \mathrm{day})$ and Sensor Medics $\operatorname{Vmax}(1700 \pm 330 \mathrm{kcal} /$ day $)(p=\mathrm{ns})$ and the correlation between REE measured by SenseWear and Sensor Medics Vmax was high $(r=0.86$, $p<0.0001)$. Bland-Altman plot showed no difference in REE determination
\end{abstract}

* Corresponding author. Tel.: +39 0592055359; fax: +39 0592055483.

E-mail address: mmalavolti@unimore.it (M. Malavolti). 
between SenseWear and Sensor Medics Vmax. \%FFM determined by BOD-POD correlated with SenseWear $(r=0.42, p<0.0001)$ as well as Sensor Medics Vmax $(r=0.38, p<0.001)$.

Conclusion: SF, BIA and BOD-POD provide valid and reliable measurements of FFM. Our results suggest that the SenseWear armband is an acceptable device to accurately measure REE in healthy subjects. Its characteristics have the potential to reduce measurement times and make the SenseWear armband useful for epidemiological studies.

(c) 2006 Elsevier B.V. All rights reserved.

\section{Introduction}

Resting energy expenditure (REE) is the largest component of total daily energy expenditure, accounting for $60-70 \%$ of total expenditure [1]. It represents, in healthy and pathological subjects, the metabolic status of body cell mass. The ability to accurately estimate REE is of the utmost importance for adequate dietary therapy. The use of metabolic carts is the standard procedure in a research setting by which REE is measured, but the equipment required to measure respiratory exchange makes this procedure timeconsuming, costly and often unavailable. To avoid this procedure and the problems related to the great variability between measurements, several prediction equations were developed $[2,3]$. The Harris-Benedict equations [3] are widely used in clinical setting and nutritional assessment. These equations, applied to a wide range of age, and body types, overestimate measured REE by at least $5 \%$ [4]. Other equations have been subsequently developed [5-7] without any improvement in the level of random error [4]. In order to avoid such errors, and to reduce the cost and complexity of this procedure, new technologies are needed to provide clinicians with more accurate and undemanding methods for measuring REE.

Recently, a new system called SenseWear ${ }^{\mathrm{TM}}$ system armband (SWA) was developed and made available on the market to assess energy expenditure. It uses sensors that continuously gather data (i.e. movement, heat flux, skin temperature, galvanic skin response) estimating the wearer's caloric expenditure, duration of physical activity and number of steps taken. Very few studies have estimated SWA validity to assess resting energy expenditure during rest and exercise $[8,9]$.

The purpose of this study was to assess the reliability and validity of the SWA, compared to metabolic cart Sensor Medics Vmax with ventilated canopy $(\mathrm{SM}-2 \mathrm{~N})$ in healthy subjects. We also assessed body composition, percentage fat mass
(\%FM) and percentage fat free mass (\%FFM) of these subjects using skinfold thickness measurements (SF), bio-electrical impedance analysis (BIA), and air displacement plethysmography (BOD-POD) and we compared \%FM and \%FFM with the results obtained by SWA and SM-29N.

\section{Methods}

\section{Subjects}

Ninety-nine healthy, normal weight subjects (52 females and 47 males), aged $38 \pm 14$ years, with body mass index (BMI) $23 \pm 3 \mathrm{~kg} / \mathrm{m}^{2}$ participated in the study. Written informed consent was obtained from all subjects and they underwent a general history and physical examination to rule out medical illnesses. Subjects were asked to follow their usual diet during the week preceding the study. All measurements were taken in the early morning after an overnight fast and subjects were instructed to void before such measurements were obtained. The institutional review boards of the University of Modena and Reggio Emilia approved the protocol.

\section{Resting energy expenditure}

REE was measured in the morning (07:00-10:00 am), using both SWA (SenseWear Pro2 Armband, BodyMedia Inc, Pittsburgh, PA, USA) and SM-29N (SM-29N Metabolic Cart, Yorbe Linda CA, USA), after a $12 \mathrm{~h}$ fast and at least $24 \mathrm{~h}$ free of structured physical activity [10]. Upon arrival at the laboratory, the SWA was placed over the triceps muscle on the upper arm of each subject. At the same time subjects were asked to rest quietly in the supine position for approximately 30$40 \mathrm{~min}$ in an isolated room, with a temperature between 21 and $24{ }^{\circ} \mathrm{C}$, after which REE measurements were performed with subjects still in the supine position. The criterion for a valid REE was 
15 min of steady state, determined as $<5 \%$ variation in respiratory quotient $(\mathrm{RQ}) /$ minute and oxygen consumption/minute. Oxygen consumption and carbon dioxide production were used to calculate REE in accordance with the Weir formula [11]. The SWA also provided a variety of measured parameters (accelerometry, heat flux, galvanic skin response, skin temperature, near body temperature) and demographic characteristics (gender, age, height, weight), entered into proprietary algorithms to estimate energy expenditure. Accelerometry is measured using a two-axis micro-electronic mechanical sensor. Heat flux is measured using a proprietary sensor that incorporates low thermal resistant materials and thermocouple arrays. Galvanic skin response is used as an indicator of evaporative heat loss and is measured by means of two hypoallergenic stainless steel electrodes. Skin temperature is used as an estimate of the body's core temperature during physical activity and is measured by a thermistor-based sensor. A body temperature sensor measures the temperature of the cover on the armband side.

As recommended by the manufacturer, the SWA was worn on the upper right arm over the triceps muscle halfway between the acromion and olecranon processes. Upon entering the laboratory, the armband was placed on the subject's arm, who wore it while seated for at least $10 \mathrm{~min}$ before data collection, to allow for acclimatisation to skin temperature. In this study, energy expenditure during each test was estimated applying a generalised proprietary algorithm (Innerview Research Software Version 4.0) developed by the manufacturer.

\section{Anthropometry}

All anthropometric measurements were performed by the same operator according to the Anthropometric Standardization Reference Manual [12]. Weight (Wt) was measured to the nearest $100 \mathrm{~g}$ and height $(\mathrm{Ht})$ to the nearest $0.1 \mathrm{~cm}$ using an electronic balance with an incorporated stadiometer (Tanita, Tokyo, Japan). BMI was calculated as $\mathrm{Wt} / \mathrm{Ht}^{2}\left(\mathrm{~kg} / \mathrm{m}^{2}\right)$.

Skinfolds and circumference were measured by a caliper and an anthropometric tape, respectively (Holtain, Crymich, UK). Skinfold thicknesses (biceps, triceps, subscapular, supra-iliac, calf and medium thigh) were measured to the nearest millimetre using calipers on the right-hand side of the body [12]. Circumferences (arm, waist, hip, calf and medium thigh) were measured to the nearest millimetre using a plastic tape measure. All skinfold and circumference measurements were repeated three times and the three values were averaged.

\section{Bioimpedance analysis}

Bioimpedance was measured using an eight-polar tactile-electrode impedance-meter (In Body 3.0, Biospace, Seoul, Korea). Body resistance (R) of arms, trunk and legs was measured in fasting subjects at frequencies of $5,50,250$ and $500 \mathrm{kHz}$ with eight-polar tactile-electrodes: two of them were in contact with the palm and thumb of each hand and the other two with the heel and the anterior part of the sole of each foot. The subjects stood with their soles in contact with the foot electrodes and grasped the hand electrodes. Further details of how the measurements were taken can be found in our previous paper [13].

Measurements were performed in the morning at room temperature $\left(21^{\circ} \mathrm{C}\right)$ after at least $12 \mathrm{~h}$ of rest, following an overnight fast.

\section{Air displacement plethysmography}

Body density was performed using air displacement plethysmography (BOD-POD, Life Measurement, Inc, Concord, CA, USA). Subjects were measured in a tight-fitting swimsuit with swim cap to minimise the effect of the hair on body volume assessment and with all jewellery removed $[14,15]$. Body mass was first measured to the nearest $0.02 \mathrm{~kg}$ on a calibrated electronic scale. Each subject was then asked to sit in the air displacement plethysmograph for body volume measurement. Subjects were instructed to sit quietly with an erect posture and normal respiration, with their hands folded in their laps and their feet placed on the floor of the device. A minimum of two 50-s tests were conducted to ensure reliability of measurements.

The body volume measurement involved two steps: (a) a standard two-point calibration process, first with the chamber empty to establish baseline values and then with a calibration cylinder $(\approx 50 \mathrm{~L})$ to establish a range of values; (b) measurement of the subject's volume in the chamber, then repeated to check for agreement. If the first two measurements were within $0.2 \%$ or $150 \mathrm{~mL}$, whichever was larger, they were averaged. If the two measurements did not meet these criteria, a third determination was made and the two values that were closest, and within the criteria for agreement, were averaged. If ambient conditions are relatively stable and the subject is breathing quietly in a relaxed way, the two measurements usually match the pre-defined criteria. 


\section{Statistical analysis}

Data were analysed using a PC version of Intercooler Stata 8 (Stata Statistical Software: Release 8.0 Collage Station, Texas). Pearson's product moment correlation analyses were performed to determine associations between REE estimate by SWA and SM-29N measurement. The Bland-Altman bias plots [16] were created to assess the agreement/difference between the indirect calorimetry measurement and SWA estimate of REE as well as the reliability of the SWA estimate. Pearson correlation was used to evaluate the relationship between \%FM and \%FFM with different body composition methods. Statistical significance was set at $p<0.05$. Data are presented as mean \pm standard deviation (SD).

\section{Results}

Physical characteristics, as well as \%FM and \%FFM of the study population are reported in Table 1. Age was not significantly different in males and females $(p=0.40)$. FFM measured by each method was higher in males than females $(p<0.0005)$. Unpaired $t$-test was computed to examine age and gender differences for the parameters measured and no statistical difference was found (all $p$ values n.s.). Therefore, all the subjects involved were analysed as a single group. REE measurements estimated by SWA $(1540 \pm 280 \mathrm{kcal} /$ day $)$ were not significantly different from those obtained by indirect calorimetry $(1700 \pm 330 \mathrm{kcal} /$ day $)(p=\mathrm{ns})$ and both methods were significantly correlated $(r=0.86$, $p<0.0001$ ). The Bland-Altman plot (Fig. 1) shows the correlation between the two measures and no bias towards over- or under-estimation.

We found a high correlation between $\mathrm{BMI}$ and REE measured by SWA $(r=0.42 ; p<0.0001)$, and between BMI and REE measured by indirect calorimetry $(r=0.40 ; p<0.0001)$.

In our sample, FFM was measured by SF, BIA and BOD-POD (Table 1) and no significant difference was detected among the different methods. The correlation coefficients between FFM measured by different techniques and REE measured by SWA and indirect calorimetry are summarised in Table 2. The best correlation was found between BODPOD FFM measurements and REE, especially FFM measured by BOD-POD which was more correlated with REE measured by SWA $(r=0.42$; $p<0.0001)$ than REE measured by SM-29N $(r=0.38$; $p<0.0001)$.

\section{Discussion}

Previous studies have been conducted to examine the validity of the SWA in order to assess energy expenditure during exercise [17], and either during exercise or after consumption of $4.8 \mathrm{~g}$ of trans-10, cis-12 conjugated linoleic acid (CLA) or olive oil capsules [9], which were thought to influence energy expenditure. In this study we examined the validity and reliability of the SWA in estimating REE compared with simultaneous indirect calorimetry measurements in normal-weight, healthy adults. No significant differences were detected. These results show that SWA could provide reliable estimates of REE. BMI was also correlated with SWA $(r=0.42, p<0.0001)$ as well as with SM-29N $(r=0.40, p<0.0001)$. According to previous studies $[18,19]$ FFM represents one of the most highly metabolically active tissues, explaining $\cong 65-90 \%$ of the variance in total energy expenditure (TEE). No significant difference was found between measurements of FFM using the different techniques. \%FFM measured by SF, BIA and BODPOD was better correlated with SWA than with SM-29N (Table 2). Above all, the best correlation was found between BOD-POD \%FFM measurements, a reliable and valid technique that can quickly and safely evaluate body composition in a wide range of subjects [20], and REE estimated by SWA $(r=0.42 ; p<0.0001)$. Data concerning REE reliability, obtained by SWA and SM-29N, were similar $(r=0.86, p<0.0001)$; such data are also similar to

Table 1 Physical characteristics of the study population

\begin{tabular}{|c|c|c|c|c|c|c|c|c|c|c|c|}
\hline & $N$ & $\begin{array}{l}\text { Age } \\
\text { (years) }\end{array}$ & $\begin{array}{l}\text { Weight } \\
\text { (kg) }\end{array}$ & $\begin{array}{l}\text { Height } \\
\text { (m) }\end{array}$ & $\begin{array}{l}\text { BMI } \\
\left(\mathrm{kg} / \mathrm{m}^{2}\right)\end{array}$ & $\% \mathrm{FM}_{1}$ & $\% F F M_{1}$ & $\% \mathrm{FM}_{2}$ & $\% F F M_{2}$ & $\% \mathrm{FM}_{3}$ & $\% \mathrm{FFM}_{3}$ \\
\hline Males & 47 & $40 \pm 6$ & $81 \pm 11$ & $1.79 \pm 0.9$ & $25 \pm 2.5$ & $20 \pm 8.0$ & $80 \pm 8.0$ & $19 \pm 5.0$ & $79 \pm 10.0$ & $20 \pm 11.0$ & $80 \pm 11.0$ \\
\hline Females & 52 & $37 \pm 13$ & $62 \pm 10$ & $1.64 \pm 0.6$ & $23 \pm 3.0$ & $29 \pm 5.6$ & $71 \pm 8.6$ & $27 \pm 6.0$ & $72 \pm 8.0$ & $27 \pm 7.0$ & $73 \pm 7.0$ \\
\hline Total & 99 & $39 \pm 15$ & $71 \pm 14$ & $1.71 \pm 0.11$ & $24 \pm 3.0$ & $24 \pm 9.0$ & $75 \pm 9.0$ & $23 \pm 6.0$ & $75 \pm 9.0$ & $23 \pm 9.0$ & $76 \pm 10.0$ \\
\hline
\end{tabular}




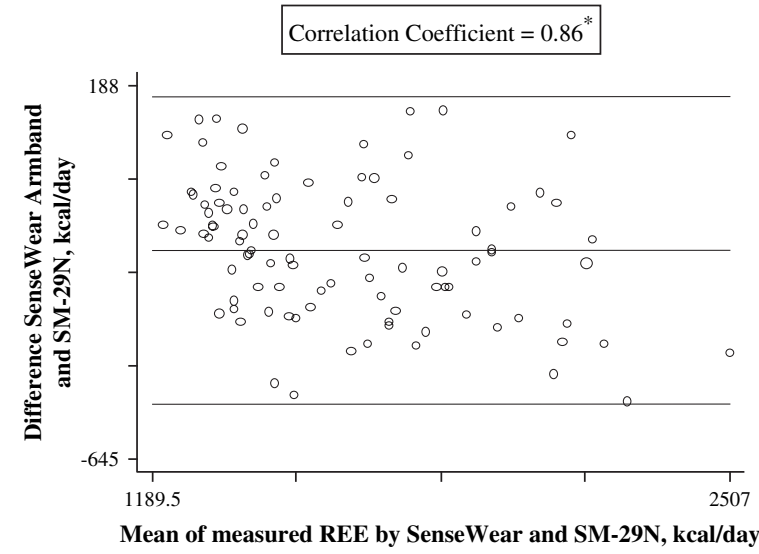

Figure 1 Bland-Altman plot to assess the difference between the SenseWear armband and Sensor Medics Vmax SM-29N $\left(N=99,{ }^{*} p=0.001\right)$ for average resting energy expenditure. The middle horizontal line represents the mean difference between the methods and the other two lines represent the $95 \%$ limits of agreement.

values reported for other indirect calorimetry systems $[21,22]$. Bland-Altman plot showed no difference in REE determination between SWA and SM-29N (Fig. 1). Fruin et al. [9] found similar results in younger subjects who, upon arrival for the resting trials, were administered CLA before the test, but our sample size was larger and with a broader age range than the above-mentioned study, and we tested the subjects according to the standard protocol [10]. We used SM-29N as the criterion for measuring SWA evaluation. However, this method should not be considered as a 'gold standard' yet, because skilled personnel are needed for its calibration and usage. Nonetheless, the metabolic cart, when used by skilled technicians, is generally considered to provide an accurate measurement of resting metabolic rate. Our results suggest that trained technicians are likely to obtain similar values for REE when using the metabolic cart and the SWA.

Table 2 Cross sectional correlation of FFM assessment estimates by SF, BIA, BOD-POD and REE measured by SWA and SM-29N

\begin{tabular}{llll}
\hline & FFM $_{\text {by SF }}$ & FFM $_{\text {by BIA }}$ & FFM $_{\text {by Bod-Pod }}$ \\
\hline REE $_{\text {by SWA }}$ & 0.20 & 0.22 & $0.42^{*}$ \\
REE $_{\text {by SM-29N }}$ & 0.19 & 0.20 & $0.36^{*}$
\end{tabular}

FFM, fat free mass; SF, skinfold thickness measurements; $\mathrm{BIA}$, bio-electrical impedance analysis; BOD-POD, air displacement plethysmography; $\mathrm{REE}_{\mathrm{by}} \mathrm{swA}$, resting energy expenditure by SenseWear system armband; REE ${ }_{\text {by }}$ SM-29N, resting energy expenditure by Sensor Medics Vmax. ${ }^{*} p<0.0001$.
The SWA method appears to have a similar accuracy and some practical advantages over traditional metabolic carts, including a significant cost reduction. SWA is portable and self-calibrating, while calibration of metabolic carts usually requires the intervention of a skilled technician. According to our results, the SWA method should increase the accessibility of REE measurements. In fact, unlike metabolic carts, SWA can be easily used by physicians and dieticians, and in many other health-care settings, while use of metabolic carts was primarily restricted to research laboratories and hospitals. Its characteristics have the potential to reduce measurement time and make the SenseWear armband useful for epidemiological studies. Finally, it is important to underline that metabolic cart measurements require standard conditions that may be obtained only in settings such as research laboratories and/or hospitals. This study confirms that the SenseWear system armband method is precise and gives accurate estimates of REE in healthy subjects. More studies are needed to further investigate this promising method and to determine its precision and accuracy in assessing REE in overweight and underweight patients.

\section{Acknowledgements}

This work was supported by SensorMedics and we also thank DS Medigroup (Milan, Italy) for supporting our study with the instruments (BOD-POD).

\section{References}

[1] Wong WW, Butte NF, Hergenroeder AC, Hill RB, Stuff JE, Smith EO. Are basal metabolic rate prediction equations appropriate for female children and adolescents? J Appl Physiol 1996;81:2407-14.

[2] Boothby WW, Sandiford RB. Normographic charts for the calculation of the metabolic rate by the gasometer method. Boston Med Surg J 1921;185:337-54.

[3] Harris JA, Benedict FG. A biometric study of basal metabolism in man. Washington, DC: Carnegie Institute of Washington; 1919. p. 40-4.

[4] Frankenfield DC, Muth ER, Rowe WA. The Harris-Benedict studies of human basal metabolism: history and limitation. J Am Diet Assoc 1998;98:439-45.

[5] Cunningham JJ. Body composition as a determinant of energy expenditure: a synthetic review and a proposed general prediction equation. Am J Clin Nutr 1991;54:963-9.

[6] Robertson JD, Reid DD. Standards for the basal metabolism of normal people in Britain. Lancet 1952;1:940-3.

[7] Schofield WN. Predicting basal metabolic rate, new standards and review of previous work. Hum Nutr Clin Nutr 1985;39(Suppl. 1):5-41.

[8] Jakicic JM, Marcus M, Gallagher KI, Randall C, Thomas E, Goss FL, et al. Evaluation of the SenseWear pro Armband 
to assess energy expenditure during exercise. Med Sci Sports Exerc 2004:897-904.

[9] Fruin ML, Rankin JW. Validity of a multi sensor armband in estimating rest and energy expenditure. Med Sci Sports Exerc 2004:1063-9.

[10] Mifflin MD, St Jeor ST, Hill LA, Scott BJ, Daugherty SA, Koh YO. A new predictive equation for resting energy expenditure in healthy individuals. Am J Clin Nutr 1990;51:241-7.

[11] Turell DJ, Alexander JK. Experimental evaluation of Weir's formula for estimating metabolic rate in man. J Appl Physiol 1964;19:946-8.

[12] Lohman TG, Roche AF, Martorell R, editors. Anthropometric Standardization Reference manual. Human Champaign IL: Human Kinetics Books; 1988.

[13] Malavolti M, Poli M, Pietrobelli A, Dugoni M, Tronfio O, Battistini NC. Body composition and nutritional habits in professional ballet dancers. Int J Body Comp Res 2005;3(2):62-8.

[14] Dempster P, Aitkens S. A new air displacement method for the determination of human body composition. Med Sci Sports Exerc 1995;27:1692-7.

[15] McCroy MA, Gomez TD, Bemauer EM, Molè PA. Evaluation of a new air displacement plethysmograph for measuring human body composition. Med Sci Sports Exerc 1995;27: 1686-91.
[16] Bland JM, Altman DG. Statistical methods for assessing agreement between two methods of clinical measurement. Lancet 1986;1:307-10.

[17] Jakicic JM, Marcus M, Gallagher KI, Randall C, Thomas E, Goss FL, et al. Evaluation of the SenseWear pro armband to assess energy expenditure during exercise. Med Sci Sports Exerc 2004:897-904.

[18] Cunningham JJ. A re-analysis of the factors influencing basal metabolic rate in normal adults. Am J Clin Nutr 1980;33:2372-4.

[19] Illner K, Brinkmann G, Heller M, Bosy-Westphal A, Muller MJ. Metabolically active components of fat free mass and resting energy expenditure in non-obese adults. Am J Physiol Endocrinol Metab 2000;278:E308-15.

[20] Fields D, Goran M, McCrory M. Body-composition assessment via air-displacement plethysmography in adults and children: a review. Am J Clin Nutr 2002;75:453-67.

[21] Weststrate JA, Weys PJ, Poortvliet EJ, Deurenberg P, Hautvast JG. Diurnal variation in post-absorptive resting metabolic rate and diet induced thermogenesis. $\mathrm{Am} \mathrm{J} \mathrm{Cl}$ Nutr 1989;50:908-14.

[22] Weststrate JA. Resting metabolic rate and diet-induced thermo-genesis: a methodological reappraisal. Am J Clin Nutr 1993;58:592-601.

Available online at www.sciencedirect.com 